\title{
Influence of Electric and Gravitational Force Fields on Nucleate Boiling of FC72 on a Wire: Results of a Sounding Rocket Campaign
}

\author{
P. Di Marco, W. Grassi \\ LOTHAR - Dipartimento di Energetica "L. Poggi" - Università di Pisa \\ Via Diotisalvi, 2 - 56126 Pisa - Italy \\ Phone+39.050.569646, Fax+39.050.830116, w.grassi@ing.unipi.it
}

\begin{abstract}
The paper reports the main results obtained during a sounding rocket experiment about the effect of gravity and electric field on nucleate pool boiling of saturated FC72 on a wire. The effect of gravitational and electric forces on the nucleate pool boiling regime have been experimentally studied. The nucleate boiling region has been found to narrow at low gravity (corresponding to a decreasing critical heat flux, CHF) and to widen thanks to the action of an imposed electric field, that increases the CHF value. Both nucleate boiling heat transfer and CHF becomes insensitive to gravity beyond a certain threshold of the applied electric field.
\end{abstract}

\section{INTRODUCTION}

A great amount of work on the influence of gravitational and electric forces on single phase convection, on the whole set of pool boiling regimes and on gas management, has been done at LOTHAR (LOw gravity and THermal Advanced Research laboratory), University of Pisa. It includes several microgravity experimental campaigns: two dropshafts at JAMIC, Japan, three (plus one more foreseen for the next autumn) aeroplane parabolic flights and two sounding rockets. A longer campaign, using the ESA facility FluidPac to be flown on the Russian satellite Foton 13, is scheduled for late 2002. The main features of the above research have been recently described in Di Marco and Grassi (2001), and the reader is referred to this paper for a rather detailed description both of the work performed and of the related bibliography.

This paper mainly reports the experimental results obtained during the sounding rocket Maser 8 campaign, held in Kiruna, Sweden, in May 1999 and concluded with the rocket launch on May 14. The availability of such a means of experimentation was a fundamental opportunity of having a quite longer period of low gravity (6 minutes compared with 5 or 10 seconds available in drop-towers and drop-shafts and the 20 seconds achievable during a parabola on an aeroplane) a low value of residual relative gravity $g / g_{0}\left(10^{-5}\right.$ instead of $10^{-2}$ of the aeroplane $)$ and a very good quality of acceleration (very small g-jitter). At present we have obtained nucleate boiling data at different levels of relative acceleration $g / g_{0}=1.5,0.4$ and 0.01 (achieved in appropriately shaped parabolic flight trajectories), $g / g_{0}$ ranging from 1.6 to around 2 during the aeroplane pull-up and pull-out flight stages, $g / g_{0}=1$ on earth and in the levelled aeroplane flight and, thanks to this last experiment, $g / g_{0}=10^{-5}$ in the sounding rocket low gravity flight stage. So far we have used three fluids: R113, FC72 (3M) and Vertrel XF (Dupont). The first and second fluids are non-polar with a relative dielectric constant equal to around 2, while the third is polar with an estimated constant around 9, but its thermophysical and electric properties are hardly available and reliable. In the following we will report and discuss the most important results obtained during the sounding rocket campaign, after a short discussion of the main features of the influence of gravitational and electric fields on pool boiling. 


\section{Influence of Electric Field on Nucleate Boiling}

The electric field affects many boiling features such as bubble shape, detachment diameter, frequency and number of nucleation sites. Also the fluid flow around the bubble, and thus the thermal field, can be modified due electrothermal convection taking place in single-phase fluids (and thus in the liquid surrounding the bubbles). Generally speaking, the volume of the detaching bubbles is a decreasing function of the dimensionless group $G_{E}=\varepsilon_{0} E^{2} l / \sigma$. The detachment volume shows also a separate dependence on $\varepsilon_{l}$ for non-polar fluids. In case of non-uniform electric fields, a net force acts on the bubbles that, for a tiny spherical bubble, can be given as $\mathbf{F}_{\mathrm{E}}=2 \pi r_{b}^{2} \frac{\varepsilon_{g}-\varepsilon_{l}}{\varepsilon_{g}+2 \varepsilon_{l}} \varepsilon_{0} \varepsilon_{l} \nabla E^{2}$.

This force tends to move bubbles towards regions with weaker electric field intensity. Thus its effect depends on how it is directed with respect to gravity. This force accelerates bubble detachment if it has the same direction as buoyancy, while the opposite occurs for e.g. downward oriented surface and electric field increasing upwards. For a cylindrical heater and a cylindrical electric field, like the one studied herein, this force tends to increase the circumferential symmetry of the boiling heat transfer and bubbles can detach also from the lower side of the heating surface. The overall effect of the electric field on nucleate boiling heat transfer is still not completely established. One can find in the literature either that there is no practical influence or that a little enhancement of the heat exchange takes place (Jones, 1978). Baboi et al. (1968) reported an increase in boiling performance at low heat fluxes. For higher fluxes, they asserted quite weakly that "there is no effect on the relative heat exchange coefficient, but on further increase of the heat flux, the latter decreases somewhat". For geometries similar to the present one, Cooper (1990) proposed a correlation providing an electric field enhancement of the heat transfer coefficient decreasing with increasing heat flux. This correlation was obtained by fitting old data sets (Baboi et al., 1968; Choi, 1962; Bonjour et al., 1962). Uemura et al. (1990) reported a little enhancement at very low heat fluxes, for saturated pool boiling of R113 on a flat, horizontal, upward facing surface. All the data (with and without electric field) collapse on a single curve for higher heat fluxes.

\section{Effect of Gravity on Nucleate Boiling}

In the absence of applied electric fields, Straub et al (1990) noted a very weak influence of gravity on pool boiling on wires. On the contrary, Oka et al (1995) reported a significant dependence on gravity of boiling heat transfer on flat plates at high heat flux; the surface overheating was also affected by the variation of direction of the residual gravity in the micro-g phase. It is worth stressing that the geometry and size of the heater (e.g. compared with the Laplace length) might play a major role on the influence exerted by the acceleration of gravity on the nucleate boiling process. For wires bubbles can leave the heater in any direction, provided that buoyancy, inertia or other "lifting forces" close to the surface are large enough. In addition any residual acceleration moves bubbles away from the heater, no matter of its direction. Therefore g-jitter helps bubbles leave the surface, with a remarkable effect on bubble motion within the fluid, but with no major effect on their behaviour once attached on the surface and thus with almost no effect on heat transfer. In fact heat transfer from a very small (compared with the Laplace length) heater "cannot sense" whether bubbles mainly detach either from its upper or its lower side.

All this does not hold for a flat sample exchanging heat with the fluid through one of its surface (upper surface on earth). The above symmetry does no longer exist and a different behaviour has to be expected.

\section{EXPERIMENTAL APPARATUS AND PROCEDURE}

The experiment consisted in performing pool boiling tests on a $0.2 \mathrm{~mm}$ Platinum wire in nearly saturated FC72 in two twin cells. These two cells were used contemporarily, one with an imposed electric field (cell A, applied high voltage of $7 \mathrm{kV}$ ) and the other without electric field (cell B). The heating sequence and the geometry were the same in the two cells, and allowed for an immediate comparison between the behaviours of these cells, thus stressing the effect of the electric forces on the process.

The experimental apparatus is shown in figure 1. It consisted of an aluminium parallelepiped vessel containing the test section. A bellows, connected to this vessel and operated by pressurised nitrogen in the secondary side, compensated for volume variations due to vapour production and thermal dilatation of the liquid. The vessel had two windows to allow for visualisation of the phenomenon by means of a video camera, with appropriate back- 


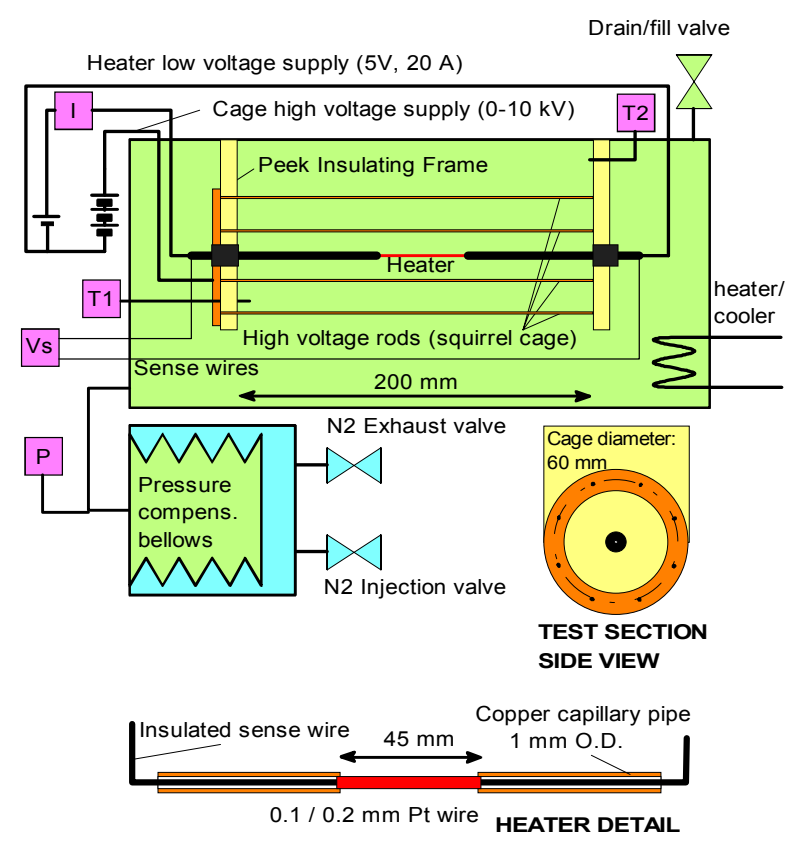

FIGURE 1. Sketch of the Experimental Set Up, Identical for Cells A and B.

illumination. Experiments were carried out using a horizontal platinum wire of $0.2 \mathrm{~mm}$ diameter and $45 \mathrm{~mm}$ length, heated by Joule effect (d.c. current) which served as both a resistance heater and a resistance thermometer. The heater (see figure 1) was made by brazing the platinum wire (the active heater) coaxial to two copper capillary tubes (1 mm OD., $0.2 \mathrm{~mm} \mathrm{ID),} \mathrm{designed} \mathrm{to} \mathrm{work} \mathrm{at} \mathrm{less} \mathrm{than} 1 \mathrm{~K}$ superheat at the maximum current rate in the experiments. Two further thin insulated wires $(0.08 \mathrm{~mm}$ diameter) were passed inside the copper tubes and brazed to the copper-platinum junctions for direct voltage sensing and measurement. This design was chosen to eliminate the distortion effects on the electric field that might arise in the presence of external sensing wires at the junctions. The use of such a small test section is due to limitations in space, power and time imposed by flight experiments constraints. A little thermal inertia is required for the test sample in order to acquire a statistically meaningful number of data in a short time.

The fluid adopted in the tests was FC72 $\left(\mathrm{C}_{6} \mathrm{~F}_{14}\right)$ a fluoroinert liquid, trademark by $3 \mathrm{M}$, slightly subcooled and ata pressure of $100 \pm 3 \mathrm{kPa}$. The electric field was produced applying a d.c. high voltage (up to $7 \mathrm{kV}$ ) between the wire and a coaxial 8-rod cylindrical "squirrel cage", of $60 \mathrm{~mm}$ diameter and $200 \mathrm{~mm}$ length. The resulting electric field configuration is discussed in (Di Marco \& Grassi, 1999).

\section{Data Acquisition And Reduction}

The acquired data for each of the two experimental cells are detailed in Table 1, together with the sampling rate and the expected accuracy. All the data were digitised via a 12 bit A/D converter.

After the experiment, the data were averaged on 16 samples (256 samples for current and voltage signals) in order to get rid of the most part of the experimental noise: this notwithstanding (see Figs. 2 and 4) some spurious spikes in the current signal, whose origin is not clear, are still present. The amplitude of these peaks has been greatly reduced by the above mentioned averaging procedure. The bulk liquid temperature in each cell was calculated as the arithmetic mean of the values provided by four sensor (AD590-M) located in the cell. The wire temperature $T_{w}$ was obtained through its electric resistance $R$, evaluated as the ratio of the voltage drop $\Delta V$ across the wire and the current $I$ flowing in the wire

$$
R=\frac{\Delta V}{I}=R_{0}\left(1+\beta T_{w}-\gamma T_{w}^{2}\right)
$$


where $R_{0}$ is the resistance at $T_{w}=0{ }^{\circ} \mathrm{C}$, and $\beta\left(\mathrm{K}^{-1}\right)$ and $\gamma\left(\mathrm{K}^{-2}\right)$ are the temperature coefficients of platinum, measured on ground before the tests on the wires mounted inside the cells. The wire superheat $\Delta T_{\text {sat }}$ and heat flux $q$ ”, exchanged with the fluid, are given as

$$
\begin{aligned}
& \Delta T_{\text {sat }}=T_{w}-T_{\text {sat }} \\
& q^{\prime \prime}=\frac{I \Delta V}{A}
\end{aligned}
$$

respectively, where $A_{h}$ is the lateral area of the wire, measured before the flight $\left(A_{h}=2 \pi r L\right)$. The value of $R_{0}, L, A_{h}$, $\beta$ and $\gamma$ for the two cells are reported in Table 2. The saturation temperature of FC72 was calculated from the value of pressure by means of the following interpolation formula (valid in the range $50-200 \mathrm{kPa}$ )

$$
T_{\text {sat }}=47.08+9.8 p-0.8 p^{2}+\frac{0.41}{p}-21.47 \ln p
$$

with $T_{\text {sat }}$ in ${ }^{\circ} \mathrm{C}$ and $p$ in bar.

TABLE 1. Acquired Experimental Data for Each of the Two Cells.

\begin{tabular}{lcc}
\hline Measured quantity & Sampling rate $(\mathrm{Hz})$ & Nominal accuracy \\
\hline Wire Current $(\mathrm{A})$ & 609.6 & $\pm 5 \mathrm{~mA}$ \\
Wire Voltage $(\mathrm{V})$ & 609.6 & $\pm 3 \mathrm{mV}$ \\
High Voltage $(\mathrm{kV})$ & 38.1 & $\pm 5 \mathrm{~V}$ \\
Temperature $1\left({ }^{\circ} \mathrm{C}\right)$ & 38.1 & $\pm 0.1 \mathrm{~K}$ \\
Temperature $2\left({ }^{\circ} \mathrm{C}\right)$ & 38.1 & $\pm 0.1 \mathrm{~K}$ \\
Temperature $3\left({ }^{\circ} \mathrm{C}\right)$ & 38.1 & $\pm 0.1 \mathrm{~K}$ \\
Temperature $4\left({ }^{\circ} \mathrm{C}\right)$ & 38.1 & $\pm 0.1 \mathrm{~K}$ \\
Bellows position $(\mathrm{mm})$ & 38.1 & $\pm 0.1 \mathrm{~mm}$ \\
Absolute pressure $(\mathrm{kPa})$ & 38.1 & $\pm 0.2 \mathrm{kPa}$ \\
\hline
\end{tabular}

TABLE 2. Characteristics of the Heating Wires of the Two Cells.

\begin{tabular}{llllll}
\hline & $\boldsymbol{R}_{\mathbf{0}}, \mathbf{m} \boldsymbol{\Omega}$ & $\boldsymbol{L}, \mathbf{m m}$ & $\boldsymbol{A}_{\boldsymbol{h}}, \mathbf{m m}^{\mathbf{2}}$ & $\boldsymbol{\beta}\left(\mathbf{K}^{-\mathbf{1}}\right)$ & $\boldsymbol{\gamma}\left(\mathbf{K}^{-\mathbf{2}}\right)$ \\
\hline Cell A & 151.2 & 44.75 & 28.12 & $3.5410^{-3}$ & $1.2610^{-6}$ \\
Cell B & 150.7 & 44.20 & 27.77 & $3.4010^{-3}$ & $3.5310^{-6}$ \\
\hline
\end{tabular}

\section{Pre-Flight Tests}

Several preliminary tests were carried out before the flight, in order to shake-down the heaters, to verify the correct operation of the control systems and to supply boiling data in normal gravity for comparison. The heating sequence consisted of a series of constant current steps and was the same as the one performed during the low gravity experiment. The test performed during Payload Test 2 phase of the hot count down (May 14) was considered the reference one for comparison, since the data were obtained in the closest conditions to the actual test under reduced gravity.

\section{TEMPORAL TREND OF THE MAIN PHYSICAL QUANTITIES IN CELL A AND B}

Figure 2 reports the trends of average bulk temperature, pressure and saturation temperature for cell A (graphs on the left) and the values of current and voltage across the heating wire (graph on the right). Time is evaluated starting from lift-off. The pressure regulation system started to operate at micro-g signals and reduced the pressure to the nominal value $(100 \pm 3 \mathrm{kPa})$ in about $10 \mathrm{~s}$. Afterwards, the pressure remained stable at a value of about $102 \mathrm{kPa}$. The heating sequence was started upon completion of the task of the pressure control system at $83 \mathrm{~s}$, i.e. about $10 \mathrm{~s}$ after the micro-g signal (start up of the low gravity phase), upon completion of the pressure regulation process. 

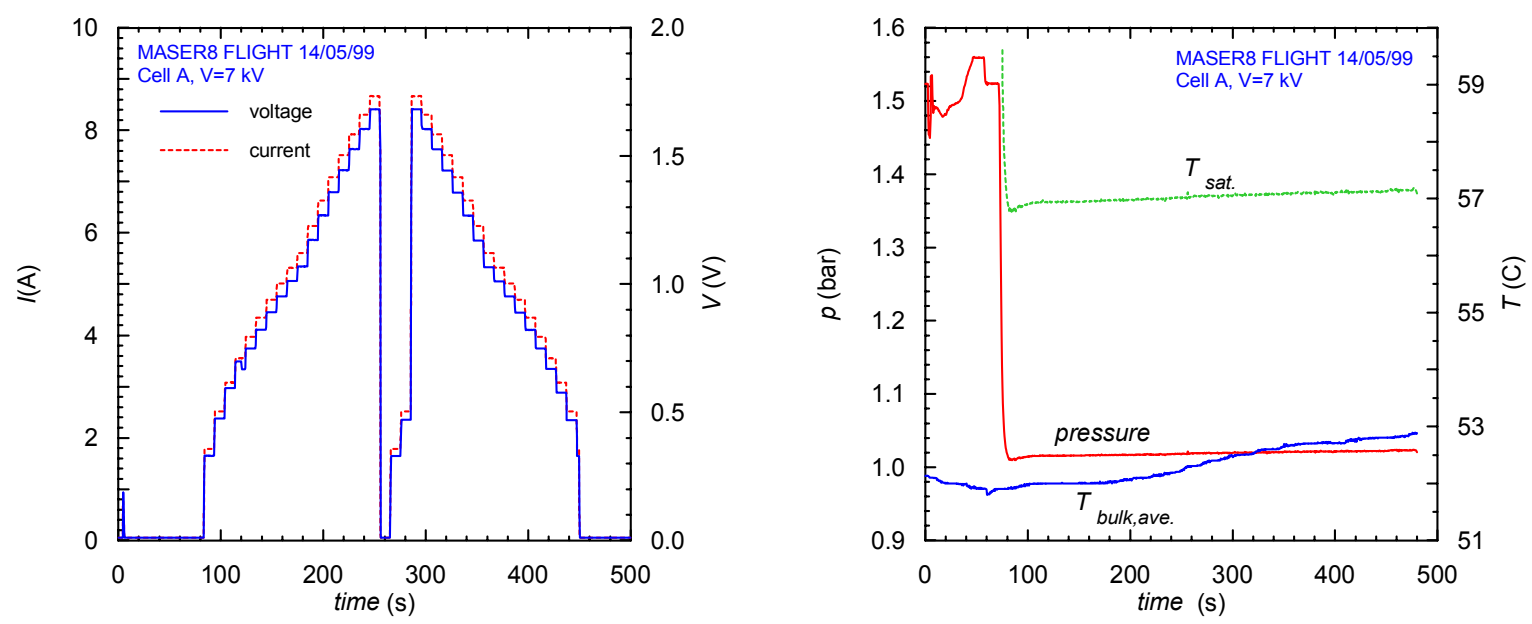

FIGURE 2. Cell A (7kV): Trends of the Main Physical Quantities vs. Time During the Flight Experiment. Graph on the Left: Bulk Pressure and Temperature and Corresponding Saturation Temperature. Graph on the Right: Current Flowing in the Test Wire and Related Voltage Drop.

It can be noted that the bulk temperature drift during the test was less than $1 \mathrm{~K}$. There was a small decrease during lift off (after the disconnection of the thermal bath, used to keep the cell bulk temperature constant before the launch) and a slight increase during the experiment, due to the heating power supplied to the test sample. The degree of subcooling was kept within 4.5 and $5.5 \mathrm{~K}$ during the whole experiment, i.e. sufficiently constant.

Figure 3 reports the trends of average bulk temperature, pressure and saturation temperature for cell B (graph on the left) as well as the values of current and voltage across the heating wire (graph on the right). Time is evaluated starting from lift-off (synchronised with cell A). The same procedure described for cell A was adopted for the pressure control. It can be noted that two further intervention of the pressure control system (at about 230 and $375 \mathrm{~s}$ ) were recorded, following the sudden production of vapour due to the onset of nucleate boiling on the wire. The operation of the pressure control system was totally satisfactory and the nominal were never exceeded. The heating sequence in cell B was restarted three times after the completion of the first one, at about 230, 340 and $440 \mathrm{~s}$. This was accomplished via the use of an appropriate telecommand from ground and allowed to obtain a repetition of the data, and in particular three almost identical values of critical heat flux. The time step was also reduced, after the first heating sequence, to $5 \mathrm{~s}$ via a dedicated telecommand.
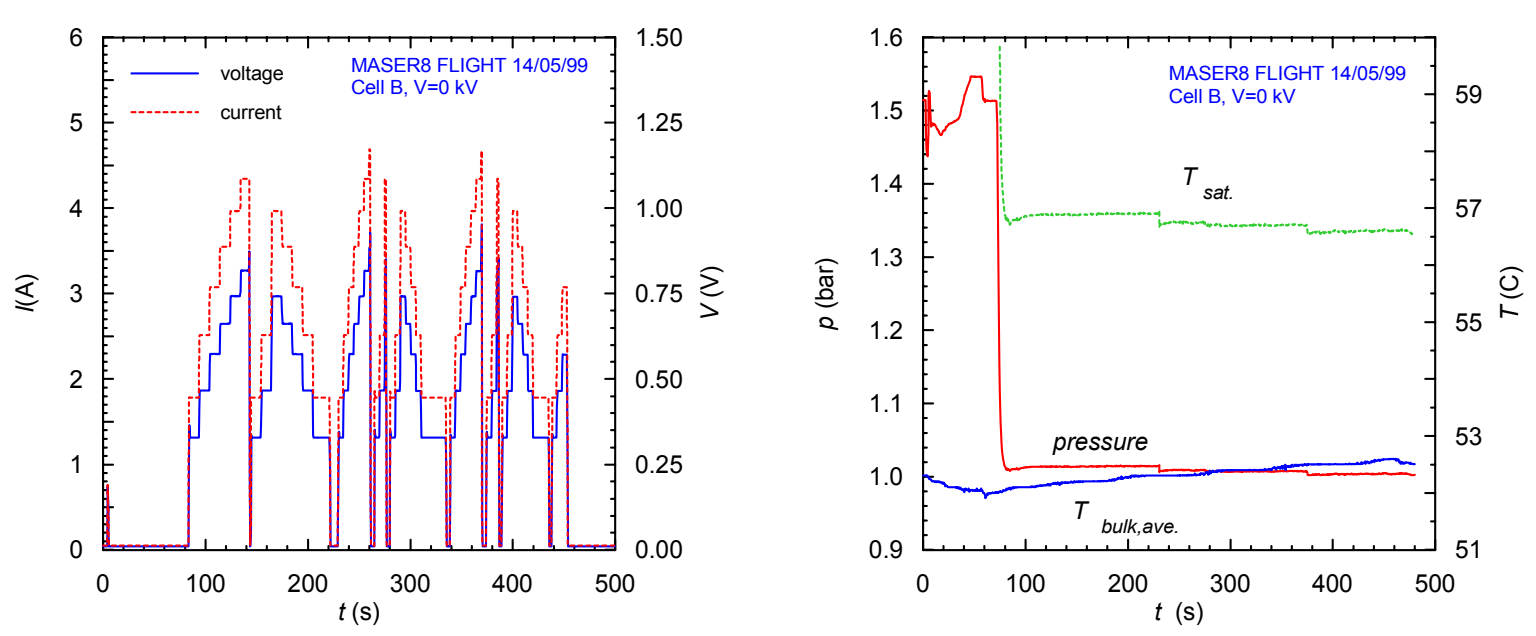

FIGURE 3. Cell B (0kV): Trends of the Main Physical Quantities vs. Time During the Flight Experiment. Graph on the Left: Bulk Pressure and Temperature and Corresponding Saturation Temperature. Graph on the Right: Current Flowing in the Test Wire and Related Voltage Drop. 


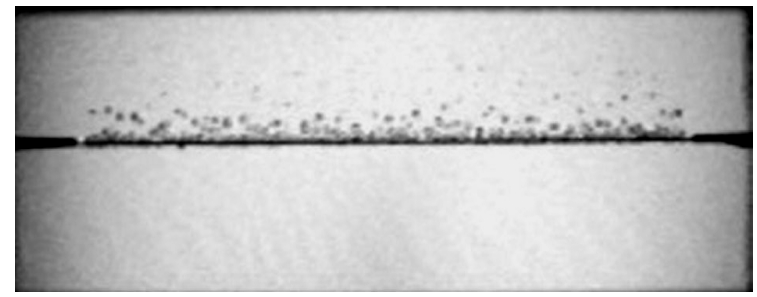

(a) Cell B (no field) $q "=130 \mathrm{~kW} / \mathrm{m}^{2}$, Nucleate Boiling at Earth Gravity.

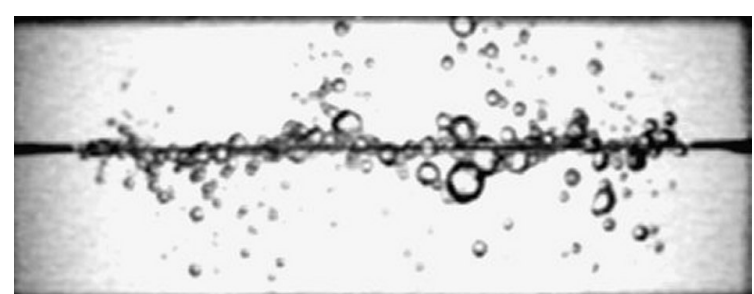

(b) Cell B (no field) $q^{\prime \prime}=130 \mathrm{~kW} / \mathrm{m}^{2}$, at $g / g_{0}=10^{-5}$, Close to CHF.

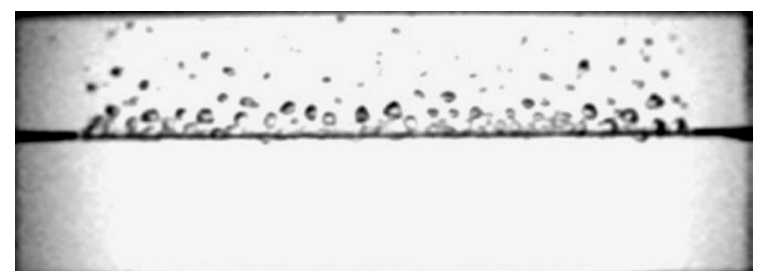

(c) Cell B (no field) $q "=210 \mathrm{~kW} / \mathrm{m}^{2}$, at Normal Gravity, Close to CHF.

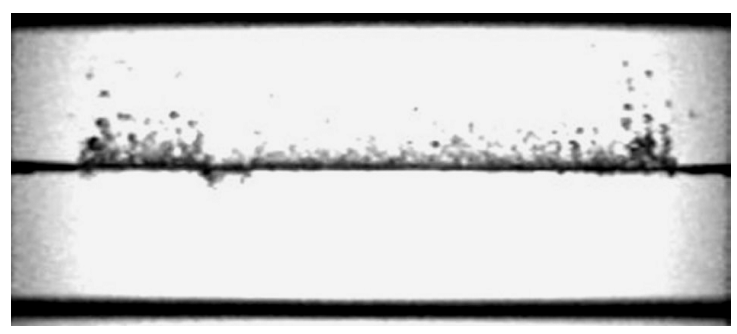

(d) Cell A (7kV) $q "=510 \mathrm{~kW} / \mathrm{m}^{2}$, Earth Gravity Close to $\mathrm{CHF}$

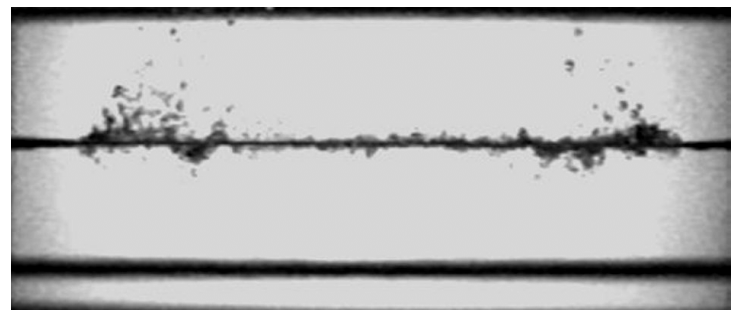

(e) Cell A ( $7 \mathrm{kV}) q^{\prime \prime}=510 \mathrm{~kW} / \mathrm{m}^{2}$, Low Gravity $\left(g / g_{0}=10^{-5}\right)$, Close to CHF

FIGURE 4. Vapour Patterns at Different Values of Gravity and Electric Field

The different (from the one adopted for cell A) heating procedure performed is due to the absence of any electric field in cell B. Owing to this the value of the critical heat flux (CHF) is consistently lower and could be reached three times during the low gravity experiment.

\section{EXPERIMENTAL RESULTS AND DISCUSSION}

The main findings on the effect exerted by electrical and gravitational (equivalent) force fields on nucleate boiling will be treated in this chapter. In particular the combined effect of both these fields will be dealt with according to the results obtained by the present authors. This effect on the vapour pattern close to the heater is shown in figure 4 . In this figure bubble size can be, at least qualitatively, appreciated with reference to the length of the boiling wire (the amperometric contacts are clearly visible on the left and right sides of the photos). Photos a, b, c refer to cell B, i.e. to the absence of electric fields. In this case the increase of bubble size due to the acceleration reduction is clearly detectable, passing from photo a to photo b. In this latter picture, the good "quality of low gravity" is testified by the fact that bubbles depart from the wire in any direction. As this behaviour persists with time it demonstrates the existence of both a low value of residual acceleration and a very small g-jitter. The above two photos refer to the same heat flux that, for earth gravity is located in the nucleate region of the boiling curve, while, in low gravity, corresponds to a condition very close to $\mathrm{CHF}$. This same condition on earth corresponds to photo c, i.e. to a much higher heat flux and to a smaller average bubble size. Conversely the other two photos ( $\mathrm{d}$ and e) refer to the presence of the electric field (cell A). Both the photos are associated to a point on the boiling curve very close to the occurrence of CHF. Bubble size is enormously reduced and is practically the same independently of the acceleration value. For this value of applied high voltage $(7 \mathrm{kV})$, the achievable critical heat flux is the same, as shown in the 
pictures' labels. An identical behaviour of the vapour pattern (big bubbles in low gravity and significant bubble size reduction due to the electric field) can be observed in the whole nucleate boiling region. This is a further confirmation of our previous observations $\left(g / g_{0}=10^{-2}\right)$ and is also in agreement with those done by other authors in low gravity without electric field and on earth with and without electric fields.

The problem of how this vapour pattern modification reflects on the heat exchange is a crucial one and has been addressed in the past by the present and by other authors, as very shortly outlined in the introduction. The results obtained during the sounding rocket flight are reported in figures 5 (a) and (b). Figure 5a refers to cell B (photos a, b, $\mathrm{c}$ of figure 4) and thus to the absence of electric field. The two curves (at normal g and in low gravity) almost coincide apart for a little shift of the low gravity curve towards higher wall superheats in correspondence of the highest heat flux. On the contrary the extension of the boiling curve is heavily influenced by gravity, in the sense that a reduction in the acceleration produces a decrease in the critical heat flux. A discussion of the influence of the electric field on CHF can be found in (Di Marco \& Grassi, 1997).

Therefore the change in the vapour pattern does not seem to exert any influence on nucleate boiling heat transfer, while it seems to be somehow correlated to the heat exchange in the region of the highest heat fluxes and to the achievable value of CHF. Figure 5b, referring to cell A (photos d and e of figure 4), describes the same situation but in the presence of the electric field. The large extension of the nucleate boiling regime caused by the application of the electric field is clearly visible and, in the present case, is the same independently of the acceleration. At the same time the two boiling curves are almost coincident, without the already discussed shift of the low gravity curve at the highest fluxes. The overall effect of the application of the electric field can be obtained by comparing the data of figure $5 \mathrm{a}$ with those in figure $5 \mathrm{~b}$. This is done in figure 6 for the results obtained in reduced gravity (a) and in normal gravity (b). Due to the practical absence of gravity figure 6a allows one to appreciate the net influence of the electric field. Beyond the already discussed enlargement of the nucleate boiling region it comes out that the boiling curve in the presence of the electric field assumes a slope larger than that of the curve obtained without field. At the same time the onset of boiling seems to be delayed and shifted towards higher wall heat fluxes. This fact might be at the origin of the previously outlined degradation of the heat exchange operated by the electric field at the lowest heat fluxes. Anyway a similar behaviour can be observed at normal gravity in figure $6 \mathrm{~b}$. The values of the applied voltage $(0$ and $7 \mathrm{kV})$ have been chosen in order to stress the phenomena as much as possible. They are somehow "extreme", in the sense that the voltage of $7 \mathrm{kV}$, in the present case, is such that the electric forces are absolutely prevailing on the gravity ones. For intermediate values of electric fields this heat transfer degradation can be followed, at higher heat fluxes by an enhancement of the heat exchange due to the field. In other words the two curves (with and without applied field), having different slopes can cross one another. This is in agreement with previous findings discussed in (Di Marco \& Grassi, 1996) for R113 and in (Di Marco \& Grassi, 1999) for FC72. The whole matter deserves some more detailed discussion not possible here for lack of space and further dedicated investigations.
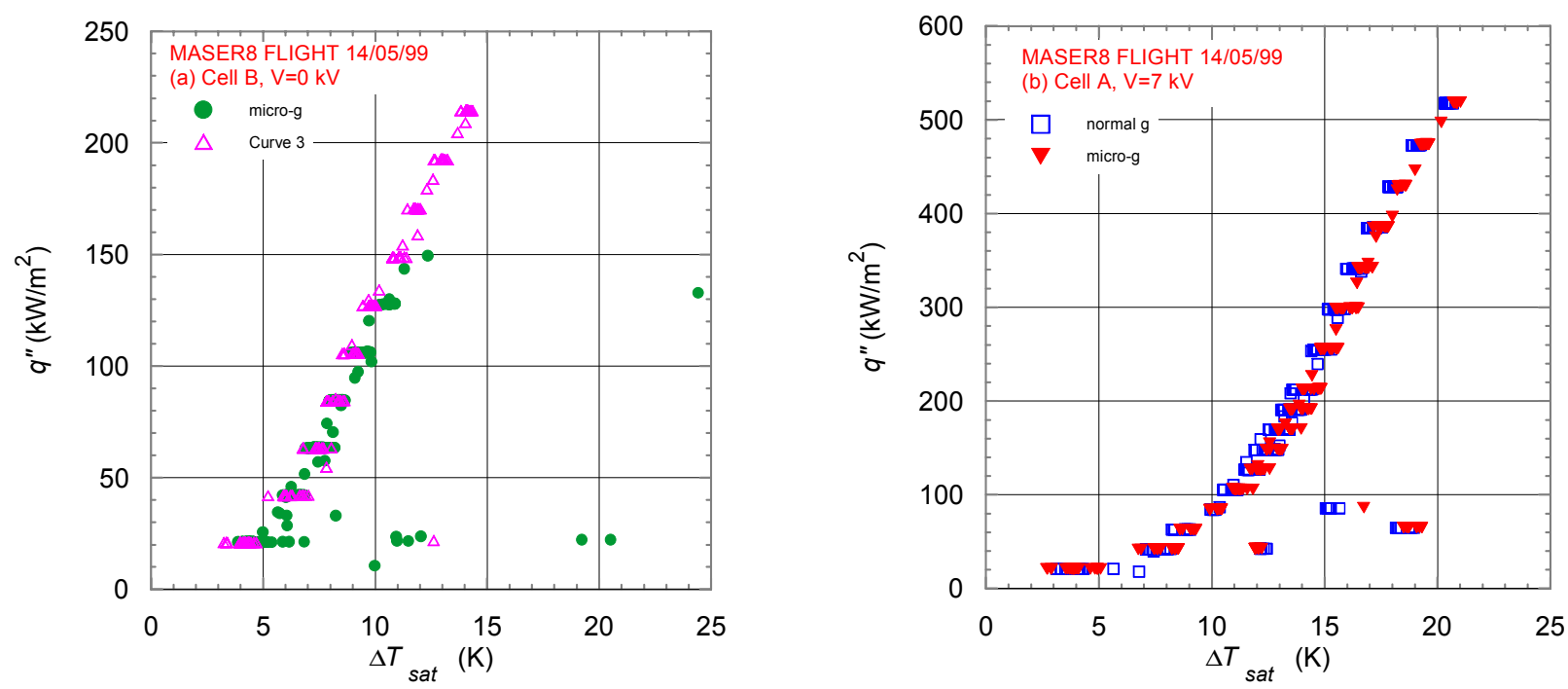

FIGURE 5. Boiling Curves for FC72 at Normal and at Reduced Gravity $\left(g / g_{0}=10^{-5}\right)$. a) In the Absence of Electric Field. b) In the Presence of an Applied High Voltage of $7 \mathrm{kV}$. 

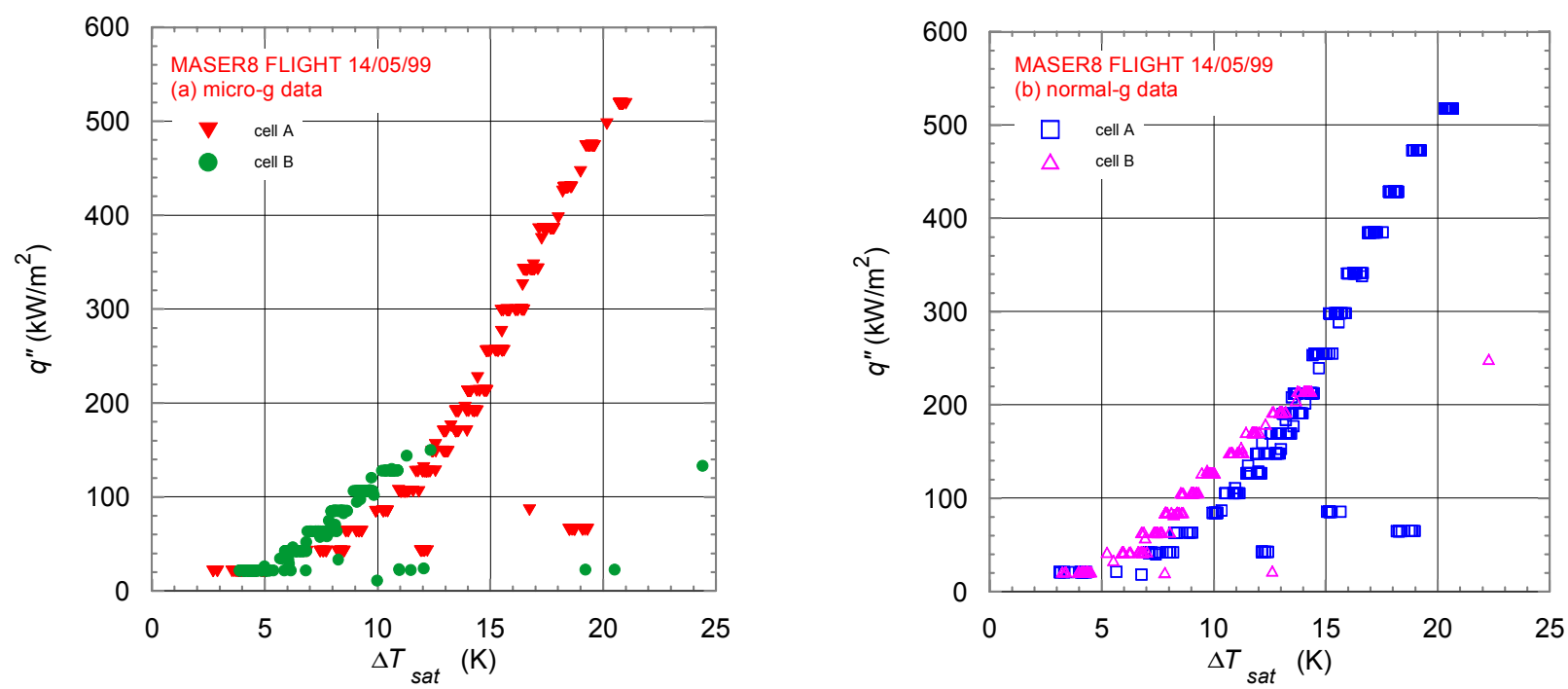

FIGURE 6. Boiling Curves for FC72 in the Absence and in the Presence of Electric Field (Applied High Voltage of $7 \mathrm{kV})$. a) At Reduced Gravity $\left(g / g_{0}=10^{-5}\right)$. b) At Normal Gravity $\left(g / g_{0}=1\right)$.

\section{CONCLUSIONS}

The influence of gravity and electric field on nucleate pool boiling on a wire immersed in almost saturated FC72 has been described in the paper, using the experimental results obtained during a sounding rocket campaign. The main conclusion that can be drawn from this study are shortly summarised as follows. Gravitational and electric forces heavily affect the vapour pattern close to the heater. A reduction in gravity determines an increase in bubble size, while the application of an electric field lowers their size and increases bubble detachment frequency. Once an appropriate electric field is applied the vapour pattern can be made insensitive to gravity changes (up to $\mathrm{g} / \mathrm{g}_{0}=10^{-5}$ ), thanks to the prevalence of the electric forces on the gravitational ones. In the absence of electric fields, the reduction of gravity does not cause any major change in the boiling heat transfer coefficient, but dramatically reduces the extension of the nucleate boiling region, owing to a reduction of the critical heat flux. The addition of an appropriate electric field enormously widens the above region (increase of CHF) and the heat transfer coefficient becomes almost insensitive to gravity. It also cause a change in slope of the curve and a delay in the boiling onset.

\section{NOMENCLATURE}

$A_{h} \quad$ heater area $\left(\mathrm{m}^{2}\right)$

$E \quad$ electric field intensity $(\mathrm{V} / \mathrm{m})$

$\mathbf{F}_{e} \quad$ electrical body force $\left(\mathrm{N} / \mathrm{m}^{3}\right)$

$g$ gravity acceleration $\left(\mathrm{m} / \mathrm{s}^{2}\right)$

$g_{0} \quad$ terrestrial gravity acceleration $\left(\mathrm{m} / \mathrm{s}^{2}\right)$

I current intensity (A)

$l \quad$ characteristic length $(\mathrm{m})$

$L \quad$ wire length $(\mathrm{m})$

$q$ " heat flux $\left(\mathrm{W} / \mathrm{m}^{2}\right)$

$R \quad$ ohmic resistance $(\Omega)$

$R_{0} \quad$ ohmic resistance at $0{ }^{\circ} \mathrm{C}(\Omega)$

$r_{b} \quad$ bubble radius $(\mathrm{m})$

$T$ temperature (K)

$\alpha \quad$ heat transfer coefficient $\left(\mathrm{W} / \mathrm{m}^{2} \mathrm{~K}\right)$

$\beta \quad$ platinum temperature coefficient $\left(\mathrm{K}^{-1}\right)$

$\gamma$ platinum temperature coefficient $\left(\mathrm{K}^{-2}\right)$
$\Delta V \quad$ voltage drop across the wire $(\mathrm{V})$

$\Delta T_{\text {sat }}$ temperature difference, $T_{w}-T_{\text {sat }}(\mathrm{K})$

$\beta \quad$ platinum temperature coefficient $\left(\mathrm{K}^{-1}\right)$

$\gamma \quad$ platinum temperature coefficient $\left(\mathrm{K}^{-2}\right)$

$\varepsilon_{0} \quad$ vacuum dielectric permittivity $(\mathrm{F} / \mathrm{m})$

$\varepsilon \quad$ relative dielectric permittivity

$\sigma \quad$ surface tension $(\mathrm{N} / \mathrm{m})$

Subscripts

CHF critical heat flux

$E \quad$ in the presence of electric field

$g$ gas

$l \quad$ liquid

sat saturation

$w \quad$ wire 


\section{ACKNOWLEDGMENTS}

The authors are indebted to Mr. R. Manetti for his skilful contribution to the experiment preparation in the laboratory. The fundamental role of Kayser Italia and Ferrari Spazio, which realised the flight equipment, and that of ESA and Swedish Space Corporation personnel, who effectively assisted the authors during the campaign, is kindly acknowledged. The flight campaign was funded by the Italian Space Agency (ASI) and by the European Space Agency (ESA).

\section{REFERENCES}

Baboi N.F., Bologa M.K., Klyukanov A.A., "Some Features of Ebullition in an Electric Field”, Appl. Electr. Phenom., (USSR), $\mathrm{n}^{\circ} 2$ (20), 1968, pp.57-70.

Bonjour E., Verdier J. and Weil L., "Improvement of Heat Exchanges in Boiling Liquids under the Influence of an Electric Field", AIChE-ASME 5th Nat. Conference, Houston (USA), 1962.

Choi L., "Electrohydrodynamic Boiling Heat Transfer", Ph.D. Thesis, MIT, Cambridge, 1962.

Cooper P., "EHD Enhancement of Nucleate Boiling", J. Heat Transfer, Trans. ASME, Vol.112, 1990, pp.458-464.

Di Marco P., Grassi W., "Nucleate Pool Boiling in The Presence of an Electric Field and in a Variable Gravity Field: Results of Experiments in Parabolic Flight", Proc. of Eurotherm Seminar n.48, ed. by D. Gorenflo, D. Kenning, C. Marvillet, Paderborn, D, 18-20 September, 1996, pp.255-264.

Di Marco P., Grassi W., "Combined Effect of Electric Field, Subcooling and Microgravity on Critical Heat Flux on a Wire in Pool Boiling", Engineering Foundation, Convective and Flow Boiling Conference, Irsee, D, 1997.

Di Marco P., Grassi W., "EHD Effects on Pool Boiling in Reduced Gravity", Proc. of the $5^{\text {th }}$ ASME/JSME Joint Thermal Engineering Conference, San Diego, CA, USA, paper AJTE99/6275, 1999a.

Di Marco P., Grassi W, "About the Scaling of Critical Heat Flux with Gravity Acceleration in Pool Boiling", Proc. XVII Congresso Nazionale UIT, Ferrara, 1999b, pp.139-149.

Di Marco P., Grassi W., "Motivation and Results of a Long-Term Research on Pool Boiling Heat Transfer in Low Gravity, Invited Lecture", Proc. of the $5^{\text {th }}$ World Conference on Experimental Heat Transfer, Fluid Mechanics and Thermodynamics, Thessaloniki, Greece, 24-28 september, 2001, pp.35-53.

Jones T.B. and Schaeffer R.C., "Electrohydrodynamically Coupled Minimum Film Boiling in Dielectric Liquids", AIAA J., Vol.14, 1976, pp.1759-1765.

Jones T.B., "Electrohydrodynamically Enhanced Heat Transfer in Liquids - A Review", Advances in Heat Transfer, Academic Press, NY., vol. 14, 1978, pp. 107-148.

Oka T., Abe Y., Mori Y.H., Nagashima A., "Pool boiling of n-pentane, CFC-113 and water under reduced gravity: parabolic flight experiments with a transparent heater", J. Heat Transfer, Trans. ASME, Vol.117, 1995, pp.408-417.

Straub J., Zell M., \& Vogel B., "Pool Boiling in a Reduced Gravity Field", Proc. 9th Int. Heat Transfer Conference, Jerusalem, Israel, KN-6, 1990, pp.91-112.

Uemura M., Nishio S., Tanasawa I., "Enhancement of Pool Boiling Heat Transfer by Static Electric Field", Proc. $9^{\text {th }}$ Int. Heat Transfer Conf., Jerusalem, Vol.4, 1990, pp.75-80. 